Service social

\title{
Un instrument de mesure pour faciliter l'évaluation et l'intervention en protection de la jeunesse
}

\section{Aline Vézina et Daniel Pelletier}

Volume 42, numéro 2, 1993

Recherche et pensée critique

URI : https://id.erudit.org/iderudit/706618ar

DOI : https://doi.org/10.7202/706618ar

Aller au sommaire du numéro

Éditeur(s)

École de service social de l'Université Laval

ISSN

1708-1734 (numérique)

Découvrir la revue

Citer cet article

Vézina, A. \& Pelletier, D. (1993). Un instrument de mesure pour faciliter l'évaluation et l'intervention en protection de la jeunesse. Service social, 42(2), 63-81. https://doi.org/10.7202/706618ar
Résumé de l'article

L'objectif général de cette étude est de faciliter l'évaluation et l'intervention en contexte de protection de la jeunesse, grâce à l'information recueillie par l'Inventaire concernant le bien-être de l'enfant en relation avec l'exercice des responsabilités parentales (ICBE). Cette information permet notamment de préciser quels alinéas des articles 38 et 38.1 de la Loi sur la protection de la jeunesse peuvent être invoqués pour statuer sur la compromission. De plus, des seuils d'intervention, c'est-à-dire des scores qui permettent d'évaluer si une intervention est requise, sont établis. 


\title{
Un instrument de mesure pour faciliter l'évaluation et l'intervention en protection de la jeunesse
}

\author{
Aline VÉZINA \\ Professeure \\ École de service social \\ Université Laval \\ Daniel PELLETIER \\ Professionnel de recherche \\ Centre de recherche sur les services communautaires \\ Université Laval
}

\section{CONTEXTE}

La pertinence et l'utilité d'instruments de mesure qui permettent de déterminer la sévérité des problèmes familiaux et les facteurs de risque pour le bienêtre des enfants ne sont pas à démontrer. Les intervenants et intervenantes aux prises avec des décisions complexes cherchent chaque jour des moyens de nuancer et d'objectiver leur jugement.

En 1986, deux auteurs américains, Steven Magura et Elizabeth Moses, ont développé, avec la collaboration de quelque 765 professionnels des services à l'enfance intervenant dans 275 organisations différentes aux ÉtatsUnis, un instrument d'évaluation du bien-être de l'enfant en relation avec I'exercice des responsabilités parentales. Cet instrument, The Child Well-Being Scales, traduit et validé au Québec sous le nom de L'inventaire 
concernant le bien-être de l'enfant en relation avec l'exercice des responsabilités parentales (ICBE), permet de faire ressortir les situations problématiques que peuvent vivre les enfants sur le plan de leurs conditions de vie au foyer, des dispositions parentales et de leur propre fonctionnement.

Le déroulement d'une première recherche menée par Vézina et Bradet en 1990 a suscité l'intérêt de professionnels de plusieurs milieux de pratique tant dans les Centres de services sociaux, aujourd'hui les Centres de protection de l'enfance et de la jeunesse, que dans les Centres locaux de services communautaires et les Centres de réadaptation. L'expérimentation de l'ICBE sur le terrain, au cours des années 1990 et 1991, démontre que pour être vraiment utiles aux intervenants et aux intervenantes, les résultats obtenus aux différentes échelles doivent être interprétés de façon plus normalisée pour faciliter les décisions d'intervention. C'est dans le but de soutenir I'utilisation de l'ICBE et de mieux cerner l'interprétation des résultats en contexte de protection de la jeunesse qu'a été réalisée l'étude de Vézina et Pelletier (1992), en étroite collaboration avec Monique Lord et Michel Thibault du Centre jeunesse de l'Estrie.

Bien que l'utilisation de l'ICBE soit accessible à tous les intervenants préalablement formés, la présente étude se situe plus spécifiquement dans le champ d'action de la protection de la jeunesse. Cela s'explique par le fait que les utilisateurs actuels de cet instrument sont surtout des intervenants en protection de la jeunesse.

\section{OBJECTIFS}

L'étude de Vézina et Pelletier (1992) vise trois objectifs, soit : 1) cibler les échelles de l'ICBE qui procurent de l'information pertinente pour statuer sur la compromission de la sécurité ou du développement de l'enfant; 2) déterminer un seuil d'intervention; et 3) proposer des mesures d'intervention. Les objectifs 1 et 2 de cette étude font l'objet du présent article. II s'agit donc, dans un premier temps, de déterminer quelles échelles de l'ICBE procurent de I'information pertinente pour statuer sur la compromission, en fonction des alinéas des articles 38 et 38.1 de la Loi sur la protection de la jeunesse. Dans un deuxième temps, des seuils d'intervention, c'est-à-dire des scores qui permettent d'évaluer si une intervention en protection de la jeunesse est requise, sont établis pour chacune des échelles.

Cette étude vise à utiliser le plus d'informations possible contenues dans I'ICBE, en gardant en mémoire que certains éléments d'évaluation et d'intervention utilisés en protection de la jeunesse ne se retrouvent peut-être pas dans I'ICBE et que I'ICBE contient peut-être des informations moins pertinentes ou moins utiles dans le contexte de la protection de la jeunesse. 


\section{QUELQUES INSTRUMENTS DE MESURE DU BIEN-ÊTRE DE L'ENFANT}

Plusieurs instruments ont été élaborés, surtout aux États-Unis, dans le but d'évaluer les problèmes familiaux chez les enfants. La plupart de ces questionnaires ne permettent pas de reconnaître tous les problèmes relatifs aux abus et à la négligence envers les enfants et de mesurer la gravité de ces problèmes. Généralement, ils ne mesurent que certains aspects du problème général ou ne s'adressent qu'à une certaine catégorie d'enfants. Par exemple, le Knowledge Scale (Epstein, 1980) mesure seulement le degré de connaissance du parent à l'égard des besoins de base et du développement (physique, moteur, perceptuel, cognitif, verbal et social) de l'enfant. Quant au Developmental Profile II (Alpern, Boll et Shearer, 1980), il comprend cinq échelles, toutes en relation avec le niveau de développement de I'enfant pour un âge donné. Le Behavior Problem Checklist (Quay et Peterson, 1979) ainsi que le Child Behavior Checklist (Achenbach, 1981) ne concernent que les problèmes de comportement chez I'enfant, alors que le Health Insurance Study (Eisen, Donald, Ware et Brook, 1980) est presque exclusivement axé sur les problèmes de santé physique, mentale et sociale. Quant au Children's Pathology Index (Alderton et Hoddinott, 1968), il est axé exclusivement sur les problèmes pathologiques de I'enfant. Le Parenting Stress Index (Abidin et Burke, 1982) a été fait dans le but de mesurer les facteurs de stress dans la relation parent-enfant. Le Family Service Association Follow-Up Questionnaire (Beck et Jones, 1980) ainsi que le Client Outcome Questionnaire (Koss, Hatry, Millar et Van Houten, 1979) mesurent davantage la perception du client quant à l'amélioration des problèmes décelés, ou encore la satisfaction du client à l'égard des services reçus, que l'importance et la gravité des problèmes constituant un risque pour la sécurité et le développement de l'enfant.

D'autres instruments ne visent qu'une certaine catégorie d'enfants. Ainsi, le Childhood Level of Living Scale (Polanski, Chalmers, Buttenwieser et Williams, 1981) s'adresse aux enfants de 1 à 7 ans et le Home Observation for Measurement of the Environment Inventory (Caldwell et Bradley, 1978) vise à mesurer le caractère stimulant de l'environnement pour le développement du jeune enfant de 0 à 6 ans.

L'instrument développé par Magura et Moses (1986), le Child WellBeing Scales, à l'encontre des autres instruments présentés précédemment, offre l'avantage de faire l'inventaire complet et détaillé des facettes concernant le bien-être de l'enfant de 0 à 18 ans, en relation avec l'exercice des responsabilités parentales. 


\section{DESCRIPTION DE L'ICBE}

L'inventaire concernant le bien-être de l'enfant en relation avec l'exercice des responsabilités parentales (ICBE) se compose de 43 échelles qui sont remplies par l'intervenant à la suite d'au moins une visite à domicile ${ }^{1}$. II importe de préciser que les parents sont définis comme le titulaire de l'autorité parentale et le conjoint légal ou de fait cohabitant au moment de l'inventaire.

Les échelles 1 à 28 concernent le fonctionnement général de la famille ou du parent par rapport à un enfant en particulier ou par rapport à tous les enfants. Avec ces échelles, l'intervenant doit indiquer un seul résultat pour la famille. Par contre, les échelles 29 à 43 exigent une évaluation différente pour chacun des enfants. Chaque échelle est composée d'une série de critères regroupés en plusieurs niveaux (de 1 à 6) partant généralement de l'adéquat (1) vers le sérieusement inadéquat (6) (voir exemple en annexe).

Dans le but d'évaluer plus précisément les situations décrites par les niveaux des différentes échelles, des scores pondérés de gravité ont été définis à l'aide de la méthode des intervalles successifs (Edwards, 1982). Sept cent soixante-cing professionnels travaillant dans le domaine de l'intervention sociale aux États-Unis ont participé à cette étape. La procédure utilisée est décrite par Magura et Moses (1986) et présentée par Vézina et Bradet (1990). Cette méthode a permis la codification des différents niveaux des 43 échelles, sur un continuum à intervalles égaux allant de 0 (sérieusement inadéquat) à 100 (adéquat).

Un score de 50 accordé pour la situation décrite au niveau 3 de l'échelle 11. Soins de santé mentale, représente le même degré de gravité qu'un score de 50 accordé pour le niveau 4 de l'échelle 15. Gestion du budget. De la même façon, l'écart entre un score de 80 et un score de 40 peut être jugé égal à l'écart entre un score de 65 et un score de 25. Cependant, il est incorrect de considérer qu'un score de 60 représente une situation deux fois plus sévère qu'une situation cotée à 30 . En effet, la valeur 0 (zéro) accordée sur une échelle à intervalles égaux ne constitue pas une absence totale de bien-être pour l'enfant. Il s'agit simplement d'un point de référence pour I'attribution des scores de gravité aux situations décrites dans les échelles. II est donc erroné de considérer ces scores comme des pourcentages (Magura et Moses, 1986).

Les scores pondérés de gravité permettent de calculer pour une famille un score moyen pour l'inventaire (ensemble des 43 échelles) ou, encore, des scores moyens pour les échelles contenues dans les trois facteurs (conditions

1. Il est important de souligner que la traduction de l'instrument, de l'anglais au français, a été réalisée par une traductrice professionnelle, en collaboration avec les chercheurs. Une double traduction du français à l'anglais n'a pu être effectuée en raison de l'ampleur de l'instrument (plus de 50 pages) et des coûts qui y étaient associés. 
de vie au foyer, dispositions parentales et fonctionnement de l'enfant) et les six dimensions théoriques (négligence physique corporelle, environnementale, émotionnelle, abus physiques, sexuels et troubles de comportement). Plus le résultat s'approche de 100, plus l'enfant vit une situation de bien-être.

L'étude de Vézina et Bradet (1990) conclut que les facteurs et les dimensions théoriques de I'ICBE obtiennent une bonne consistance interne mesurée à l'aide du coefficient Alpha. Ces coefficients varient entre 0,66 et 0,87. Pour ce qui est de la stabilité test re-test, pour un intervalle moyen de 2,8 semaines, les corrélations intra-classes varient entre 0,56 et 0,82 . L'ICBE offre une bonne validité de convergence et de concomitance. De plus, les résultats à l'instrument ne sont pas affectés par les caractéristiques de l'intervenant qui l'utilise, tels le nombre de mois ou d'années d'affectation au dossier, le nombre de visites effectuées au domicile, le nombre d'années d'expérience, le sexe, l'âge et la scolarité (validité de différenciation).

\section{MÉTHODE}

\section{Sujets}

Afin d'atteindre les objectifs de l'étude, deux catégories de sujets sont retenues. Ainsi, pour déterminer les échelles de I'ICBE qui procurent de I'information pertinente relativement aux alinéas des articles 38 et 38.1 de la Loi sur la protection de la jeunesse, la participation de juges experts est sollicitée. Est considéré comme juge expert, tout intervenant ou gestionnaire ayant suivi la formation à l'utilisation de l'ICBE et possédant une expérience clinique de plus de cing ans dans I'application de la Loi sur la protection de la jeunesse et dans la problématique diverse qu'on y rencontre. Au moment de la recherche, seuls les intervenants des Centres de protection de l'enfance et de la jeunesse (CPEJ) du Bas-du-Fleuve, de l'Estrie, de Laurentides-Lanaudière et de Québec avaient reçu la formation à l'utilisation de l'instrument.

Onze juges experts, quatre hommes et sept femmes, sont retenus. Trois viennent du CPEJ de I'Estrie, trois des Laurentides-Lanaudière, deux du Basdu-Fleuve, un de Québec, un de l'Outaouais ${ }^{2}$ et un d'un centre de réadaptation pour mères en difficulté, la Villa Marie-Claire de Sherbrooke. Tous les juges experts avaient une formation universitaire en service social du niveau baccalauréat ou maîtrise.

Les 11 juges experts ainsi que 27 utilisateurs ont permis de déterminer les seuils d'intervention des 43 échelles de l'ICBE. Les utilisateurs qui ont

2. Le juge expert du CPEJ de l'Outaouais avait suivi une formation à l'utilisation de I'ICBE dans un autre CPEJ. 
participé à l'étude sont des intervenants qui ont reçu une formation à l'ICBE conforme aux données de validation, d'utilisation et d'interprétation présentées dans l'étude de Vézina et Bradet (1990). La répartition des 27 utilisateurs selon les différents CPEJ est la suivante: six pour le CPEJ de l'Estrie, trois pour le CPEJ de Québec, sept pour le CPEJ Bas-du-Fleuve et 11 pour le CPEJ Laurentides-Lanaudière. Parmi les 38 participants, juges experts et utilisateurs, se trouvent 23 femmes et 15 hommes. La moyenne d'âge est de 37,8 ans. Les formations scolaires sont variées.

Il est important de noter que 38 participants apparaît un nombre satisfaisant compte tenu du critère "avoir suivi la formation », qui réduit considérablement le nombre de sujets admissibles pour l'étude, et du temps de réalisation de la tâche qui s'additionnait à une charge de travail passablement lourde chez les intervenants et gestionnaires de la protection de la jeunesse. Il s'agit donc d'un échantillon non probabiliste de volontaires (Trudel et Antonius, 1991).

\section{Procédure}

Afin de déterminer les échelles de l'ICBE qui procurent de l'information pertinente par rapport aux alinéas des articles 38 et 38.1 de la Loi sur la protection de la jeunesse, les juges experts répondent individuellement à un questionnaire en appliquant la consigne suivante:

En passant systématiquement en revue les différentes composantes de l'ICBE, cochez quelles échelles (aucune, une ou plusieurs), vous associez directement à chacun des alinéas des articles 38 et 38.1 présentés dans le Manuel de référence sur la Loi sur la protection de la jeunesse (MSSS, 1990).

Afin de préciser les seuils d'intervention, les 38 participants, juges experts et utilisateurs, répondent individuellement à un questionnaire à l'aide de la consigne suivante:

En reprenant les échelles de l'inventaire une à une, déterminez le niveau (c'est-à-dire de 1 à 6) à partir duquel vous jugez qu'une intervention en protection de la jeunesse sera couramment requise.

\section{RÉSULTATS}

Le tableau 1 présente les échelles de l'ICBE retenues par $70 \%$ et plus des juges experts comme procurant de l'information pertinente par rapport aux alinéas des articles 38 et 38.1 de la Loi sur la protection de la jeunesse. Le pourcentage retenu est arbitraire et ne vise qu'à conserver les échelles les plus pertinentes. 
TABLEAU 1

Échelles de I'ICBE jugées par $70 \%$ des experts comme procurant de I'information pertinente par rapport aux alinéas des articles 38 et 38.1 de la Loi sur la protection de la jeunesse $(\mathrm{N}=11)$

ALINÉAS DE LA L.P.J.
ÉCHELLES RETENUES PAR $70 \%$ ET PLUS

DES JUGES EXPERTS

\begin{abstract}
Abandon : 38 a)
Si ses parents ne vivent plus, ne s'en occupent plus ou cherchent à s'en défaire

\section{Menace au développement mental} ou affectif : $\mathbf{3 8}$ b)

Si son développement mental ou affectif est menacé par l'absence de soins appropriés ou par l'isolement dans lequel il est maintenu ou par un rejet affectif grave et continu de la part de ses parents
\end{abstract}

Menace à la santé physique : 38 c)

Si sa santé physique est menacée par l'absence de soins appropriés
18 Continuité de la figure parentale

24 Acceptation et manifestation d'affection

11 Soins de santé mentale

16 Capacités parentales à assurer les soins à I'enfant

24 Acceptation des enfants et manifestation d'affection

25 Approbation des enfants

27 Cohérence de la discipline au foyer

1 Soins de santé physique

2 Nutrition/ régime alimentaire

3 Habillement

4 Hygiène personnelle

7 Propreté et entretien de la maison

10 Sécurité physique à la maison

12 Surveillance des jeunes (- de 12 ans)

\section{Privation de conditions matérielles}

d'existence : $38 \mathrm{~d}$ )

S'il est privé des conditions matérielles d'existence appropriées à ses besoins et aux ressources de ses parents ou de ceux qui en ont la garde
16 Capacités parentales à assurer les soins à I'enfant

30 Privation physique délibérée (du boire et du manger)

2 Nutrition/ régime alimentaire

3 Habillement

5 Ameublement dans la maison

6 Surpeuplement de la maison

7 Propreté et entretien de la maison
Risque de danger moral ou physique : 38 e)

S'il est gardé par une personne dont le comportement ou le mode de vie risque de créer pour lui un danger moral ou physique
8 Assurance d'un lieu de résidence

9 Services disponibles dans la maison

10 Sécurité physique à la maison

15 Gestion du budget

14 Gardiennage

16 Capacités parentales à assurer les soins à I'enfant 


\section{Tableau 1 (suite)}

ALINÉAS DE LA L.P.J.
ÉCHELLES RETENUES PAR $70 \%$ ET PLUS

DES JUGES EXPERTS

\section{Exploitation : $38 \mathrm{f}$ )}

S'il est forcé ou incité à mendier, à faire un travail disproportionné à ses capacités ou à se produire en spectacle de façon inacceptable eu égard à son âge

Mauvais traitement physique : $\mathbf{3 8} \mathbf{g}$ ) $S^{\prime}$ il est soumis à des mauvais traitements physiques par suite d'excès ou de négligence

\section{Abus sexuel : $38 \mathbf{g}$ )}

S'il est victime d'abus sexuels

\section{Troubles de comportement sérieux : $38 \mathrm{~h}$ )}

S'il manifeste des troubles de comportement sérieux et que ses parents ne prennent pas les moyens nécessaires pour corriger la situation ou n'y parviennent pas

\section{Fugue : 38.1 a)}

$S^{\prime}$ il quitte sans autorisation son propre foyer, une famille d'accueil ou un centre hospitalier alors que sa situation n'est pas prise en charge par le directeur de la Protection de la jeunesse

\section{Absentéisme scolaire : $\mathbf{3 8 . 1}$ b)}

$S^{\prime}$ il est d'âge scolaire et ne fréquente pas l'école ou s'en absente fréquemment sans raison

\section{Les parents ne s'acquittent pas de leurs obligations : $38.1 \mathrm{c}$ )}

Si ses parents ne s'acquittent pas des obligations de soin, d'entretien et d'éducation qu'ils ont à l'égard de leur enfant ou ne s'en occupent pas d'une façon stable, alors qu'il est confié à un établissement ou à une famille d'accueil depuis deux ans
17 Relations entre conjoints

18 Continuité de la figure parentale

19 Reconnaissance du problème par les parents

20 Motivation des parents à résoudre le problème

36 Protection contre abus, $1^{\text {re }}$ entrevue

35 Exploitation économique

29 Discipline physique abusive

30 Privation physique délibérée (du boire et du manger)

31 Restrictions physiques excessives

33 Abus sexuels, parent + autre

34 Menaces d'abus

19 Reconnaissance du problème par les parents 20 Motivation des parents à résoudre le problème

41 Problème de conduite de l'enfant

42 Comportement d'adaptation de l'enfant
(Aucune échelle)

37 Besoins d'instruction

39 Fréquentation scolaire

21 Coopération des parents avec les services

Il se dégage à la lecture du tableau 1 que sur la base d'un taux d'accord de $70 \%$ entre les juges experts, l'ICBE ne procure pas d'information pertinente en rapport avec l'alinéa 38.1 a) de la Loi sur la protection de la jeunesse (la 
fugue). De plus, une seule échelle procure de l'information pour l'alinéa 38 f) (I'exploitation) et 38.1c) (les parents ne s'occupent pas de leurs obligations). Il est intéressant de remarquer que de façon générale les échelles pertinentes à considérer dans I'ICBE sont différentes d'un alinéa à l'autre. En d'autres mots, peu d'échelles reviennent plus d'une fois ou deux dans le tableau. Cette spécificité des échelles constitue une qualité importante de l'ICBE dans le sens où les échelles mesurent bien ce qu'elles doivent mesurer et, de ce fait, sont mutuellement exclusives.

Avant d'aborder plus directement les seuils d'intervention, certains constats s'imposent. Il est essentiel de mentionner tout d'abord que les résultats obtenus pour chacune des 43 échelles, c'est-à-dire le niveau (de 1 à 6) à partir duquel une intervention en protection de la jeunesse sera couramment requise, varient très peu parmi les 38 sujets. Ainsi, pour 31 échelles sur 43 , les résultats de $90 \%$ et plus des sujets se répartissent sur seulement deux niveaux; pour 6 échelles sur 43, 90\% des sujets et plus ont choisi le même niveau. À titre d'exemple, pour l'échelle 13. Supervision des adolescents, 34 participants sur $38(90 \%)$ ont choisi le niveau 4. Pour l'échelle 20. Motivation des parents à résoudre les problèmes, 36 sujets sur 38 (95\%) ont choisi le niveau 3 ou le niveau 4. Cette faible variance dans la distribution des résultats de l'ensemble des sujets est très intéressante. Elle indique, notamment, que la majorité d'entre eux partagent à peu près les mêmes critères d'évaluation en ce qui concerne la gravité d'une situation nécessitant une intervention en protection de la jeunesse. De plus, ces résultats montrent que les 43 échelles du bien-être de l'enfant en relation avec l'exercice des responsabilités parentales sont mesurées à partir d'indicateurs de gravité définis de façon claire et opérationnelle, ce qui laisse peu de place à la subjectivité.

Afin de déterminer si les résultats varient entre les juges experts et les utilisateurs, un score moyen ${ }^{3}$ à l'ICBE pour chaque sous-groupe est calculé, en utilisant les scores pondérés de gravité associés aux niveaux identifiés comme requérant une intervention en protection de la jeunesse. Le score moyen est de 43,15 pour les juges experts et de 44,8 pour les utilisateurs. Cette différence n'est pas significative. En effet, selon la table de la distribution de Student disponible dans Trudel et Antonius (1991), pour un degré de liberté se situant entre 30 et 40, une différence de 1,684 et plus est nécessaire entre les deux moyennes pour qu'elle soit significative à $p<0,05$. La différence est ici de 1,65.

3. Le score moyen à l'ICBE, pour chaque sujet, est calculé en additionnant les scores pondérés de gravité des 43 échelles divisé par 43. La somme des scores moyens des 38 sujets divisée par 38 donne le score moyen pour l'ensemble de l'échantillon. La somme des scores moyens des 11 juges experts divisée par 11 donne le score moyen pour les juges experts et ainsi de suite. 
En plus d'un score moyen à l'ICBE pour les juges experts et les utilisateurs, un score moyen pour chacun des quatre CPEJ retenus pour l'étude est calculé. Les résultats sont les suivants: Québec 42,4, Estrie 42,4, Laurentides-Lanaudière 45,0 et Bas-du-Fleuve 45,8 . Le très petit nombre de sujets par CPEJ, particulièrement celui de Québec (trois utilisateurs et un juge expert), ne permet pas d'effectuer des analyses statistiques fiables. Mentionnons que les CPEJ de l'Estrie et de Québec obtiennent le même score et les CPEJ Laurentides-Lanaudière et Bas-du-Fleuve des scores presque identiques. Bien qu'un test de Fisher (analyse de variance) soit nécessaire pour appuyer nos dires (Gilbert et Savard, 1978), il ne semble pas y avoir une différence assez importante entre les moyennes des quatre groupes pour motiver le regroupement des résultats selon le CPEJ d'appartenance. Ainsi, pour la présentation

\section{Figure 1}

Seuils d'intervention pour les 43 échelles de l'ICBE $(\mathrm{N}=38)$
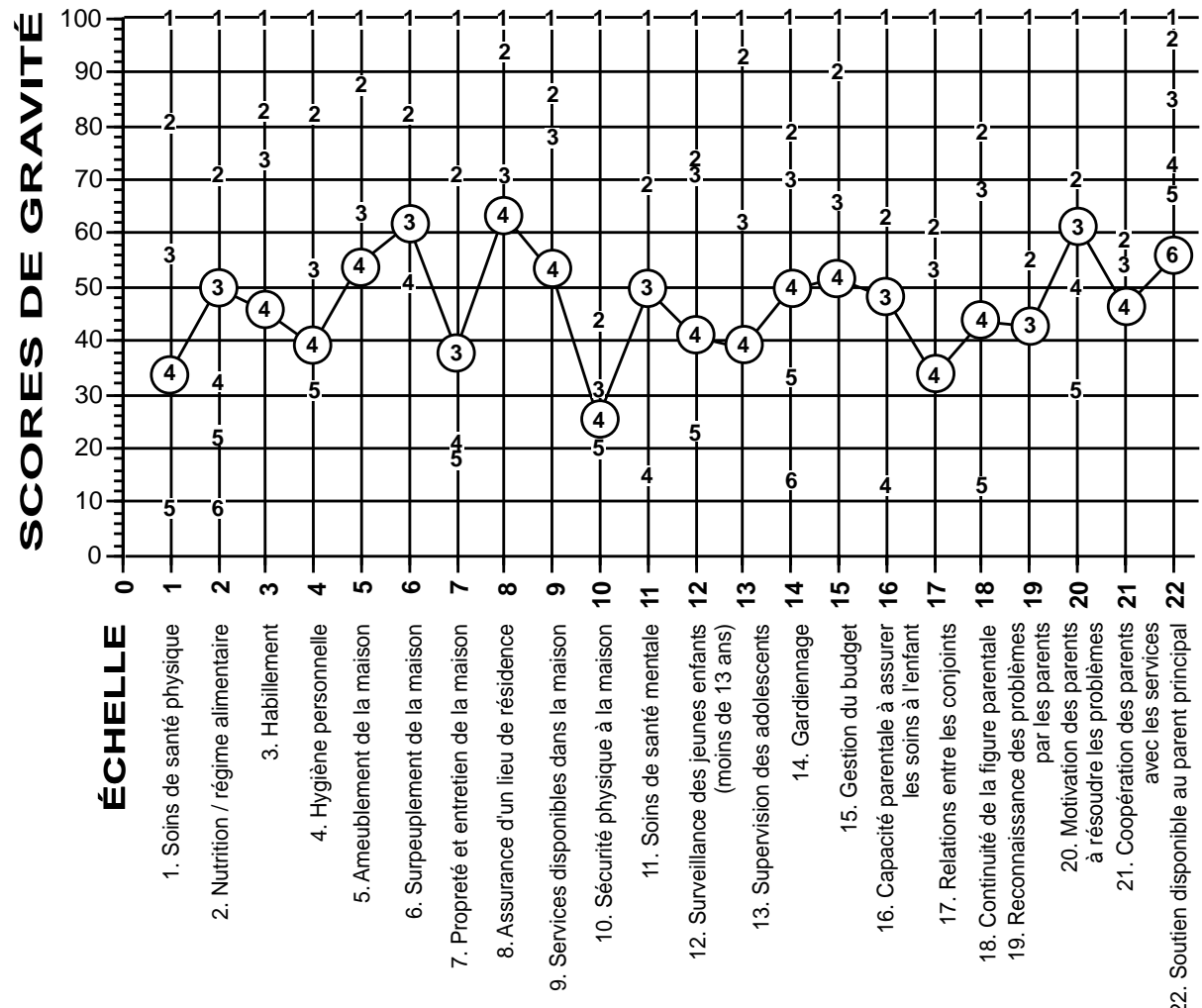

* Plus de $50 \%$ des sujets ont indiqué ne pas considérer l'échelle 23. Disponibilité et accessibilité des services et l'échelle 38. Rendement scolaire pour juger qu'une intervention en protec- 
des résultats, les calculs sont effectués pour l'ensemble des 38 participants de l'étude.

Rappelons brièvement que pour chacune des 43 échelles, les juges experts et les utilisateurs doivent déterminer le niveau (de 1 à 6) à partir duquel une intervention en protection de la jeunesse est couramment requise. Les niveaux des échelles progressent de 1 (très adéquat) à 6 (sévèrement inadéquat). Les résultats sont analysés à l'aide du mode. Le mode est le niveau identifié par le plus grand nombre de sujets.

La figure 1 présente les seuils d'intervention pour chacune des 43 échelles. Il est important de noter que les niveaux ont été transformés en scores pondérés de gravité ainsi qu'il est décrit dans Magura et Moses (1986) et Vézina et Bradet (1990). Mentionnons de plus que les échelles 33 et 36

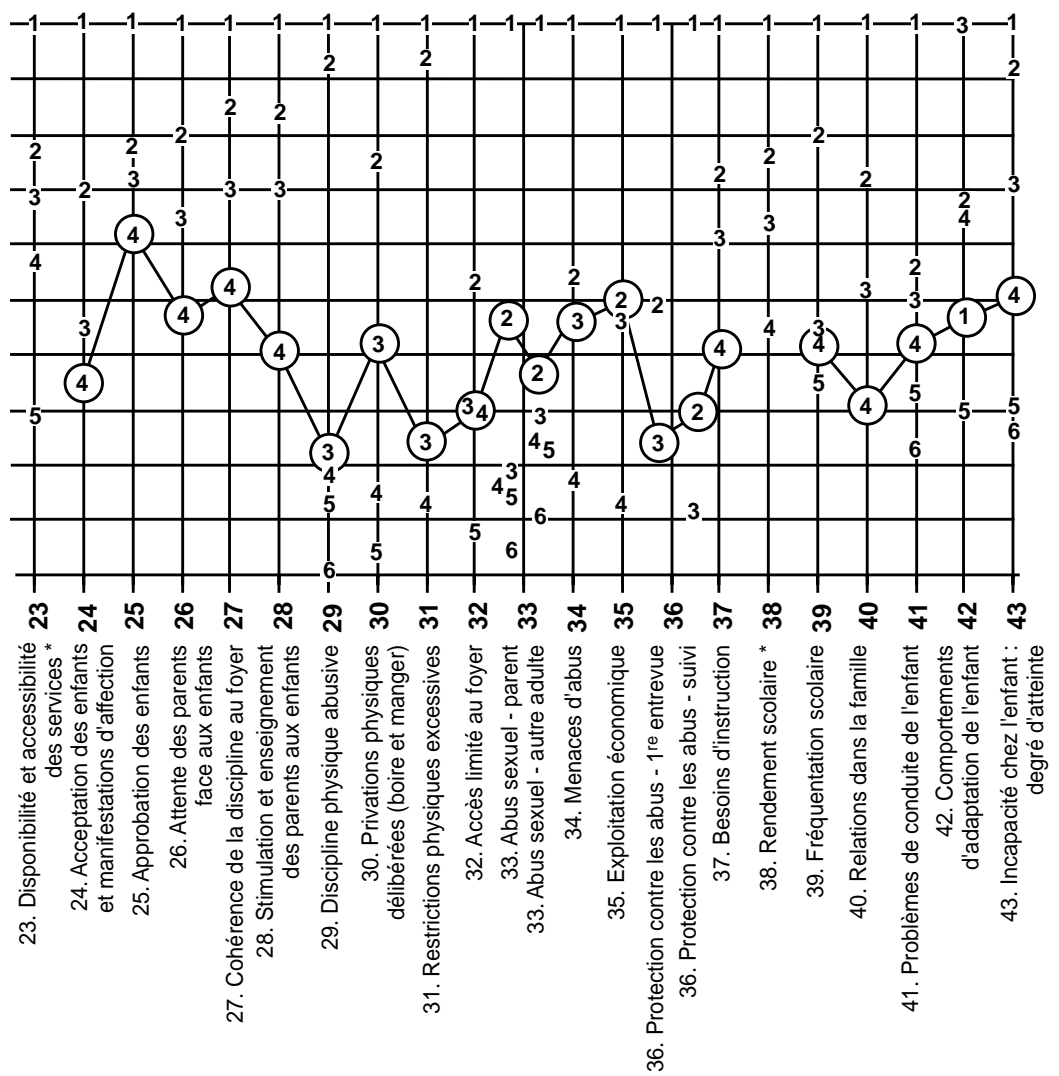

tion de la jeunesse sera couramment requise. En raison de ce grand nombre de données manquantes, les seuils d'intervention de ces deux échelles n'ont pas été déterminés. 
obtiennent deux seuils d'intervention. Il s'agit d'un seuil d'intervention pour 33. a Abus sexuel, commis par le parent, 33.b Abus sexuel, commis par un autre adulte; 36. a Protection contre les abus, $1^{\text {re }}$ entrevue et 36.b Protection contre les abus, suivi. Les seuils d'intervention pour les échelles 23. Disponibilité et accessibilité des services et 38. Rendement scolaire n'ont pu être calculés du fait que $50 \%$ et plus des participants ont mentionné ne pas considérer ces échelles pour juger qu'une intervention en protection de la jeunesse est requise. Les seuils d'intervention pour chacune des échelles sont regroupés selon les facteurs à la figure 2 et selon les dimensions théoriques à la figure 3.

\section{UTILISATION ET INTERPRÉTATION DES RÉSULTATS}

L'utilisation et l'interprétation des résultats obtenus à l'ICBE se présentent sous forme d'étapes ou de phases dont l'ordre peut être changé en toute liberté. La présentation de ces étapes, bien que linéaire (c'est-à-dire que les étapes se réalisent selon une séquence définie), n'exclut nullement la possibilité que I'intervenant revienne, au cours du processus d'évaluation, vers des étapes antérieures. Afin de mieux comprendre et de mieux intégrer les résultats présentés dans cette étude, la démarche proposée débute au moment où I'intervenant veut évaluer la situation d'un enfant et de sa famille à l'aide de I'ICBE.

\section{Observation de la situation}

Dans un premier temps, I'intervenant doit recueillir l'information nécessaire, inscrire la cote appropriée de 1 à 6 pour chacune des 43 échelles de l'ICBE et changer les cotes en scores pondérés de gravité. Pour se donner une information synthèse, il peut effectuer les calculs du score moyen total et des scores moyens correspondant aux facteurs et dimensions théoriques ${ }^{4}$.

Afin de dresser le profil détaillé de la situation de l'enfant et de sa famille, il reporte les résultats à chacune des 43 échelles de l'ICBE sur la grille des scores pondérés de gravité présentée à la figure 1. Le profil détaillé des 43 échelles de l'ICBE permet de repérer d'un seul coup d'œil les facettes où le bien-être de l'enfant est menacé (les scores tendent vers 0 ) et celles où le bien-être est assuré dans son milieu familial (les scores tendent vers 100). L'intervenant peut aussi vouloir regrouper les échelles qui représentent des secteurs importants du bien-être de l'enfant. II reporte alors les scores obtenus

4. Pour plus d'informations concernant la cotation de l'ICBE et le calcul des scores moyens, consulter l'étude de Vézina et Bradet (1990). 
Figure 2

Seuils d'intervention pour les facteurs de I'ICBE $(\mathbf{N}=38)$
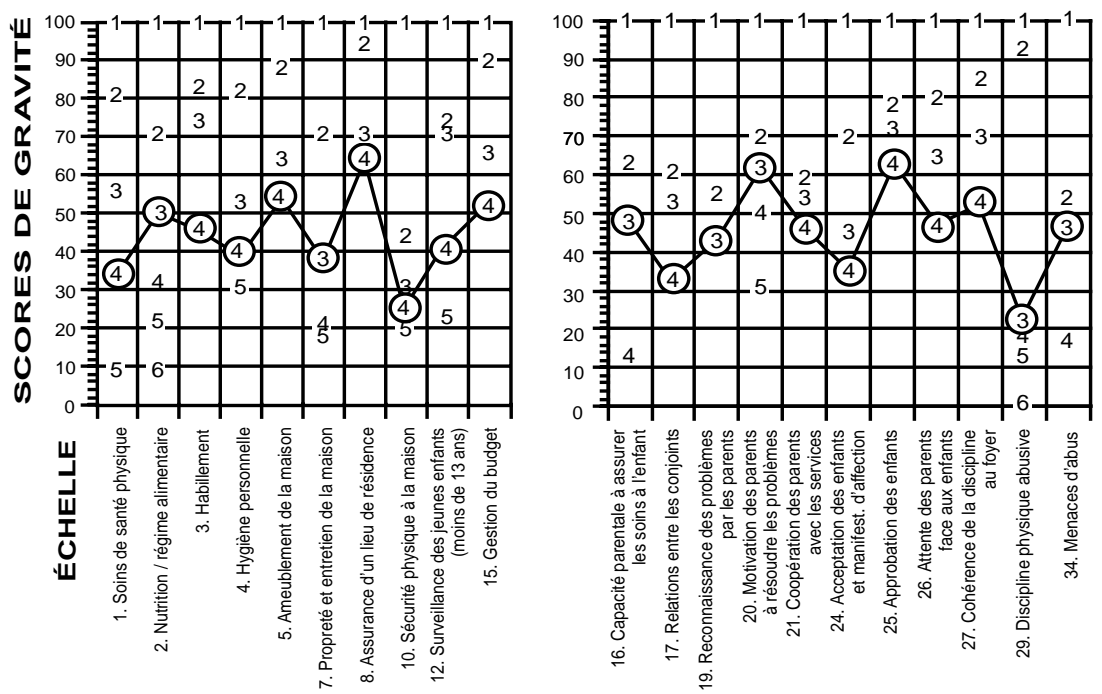

Facteur 1 : Conditions de vie au foyer

Facteur 2 : Dispositions parentales

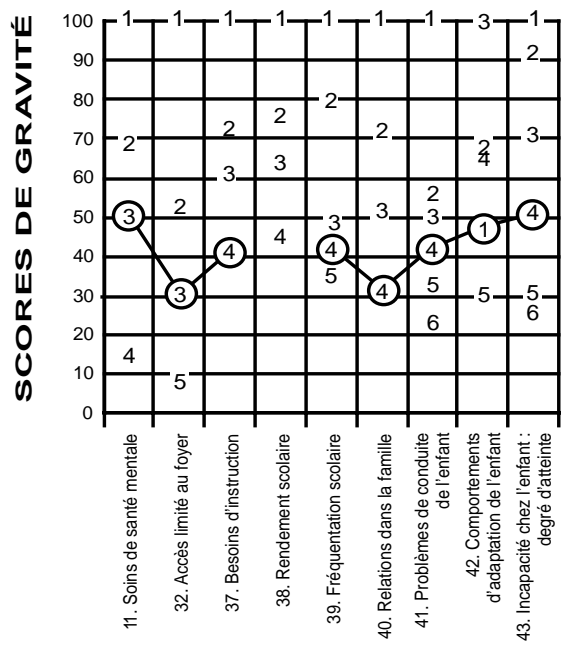

Facteur 3 : Fonctionnement de l'enfant 
Figure 3

Seuils d'intervention pour les dimensions théoriques de I'ICBE $(N=38)$

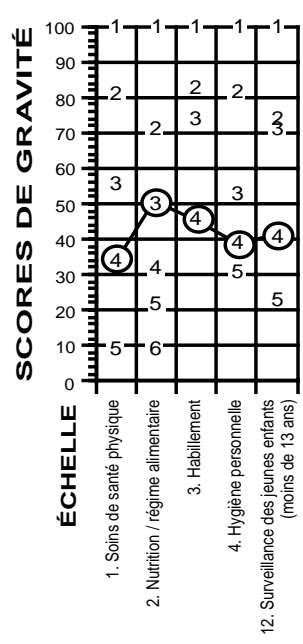

Négligence physique corporelle
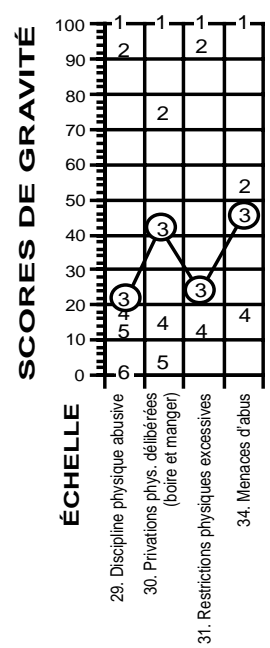

Abus physiques
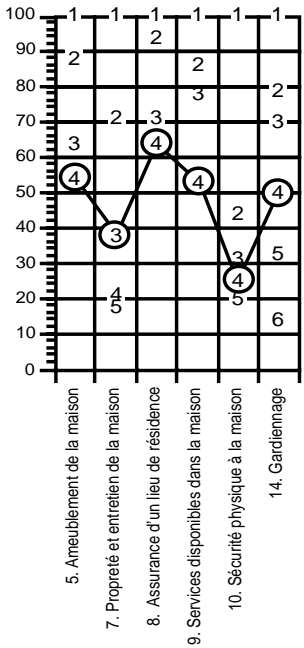

$$
\begin{gathered}
\text { Négligence } \\
\text { physique } \\
\text { environnementale }
\end{gathered}
$$

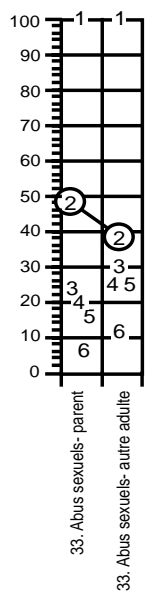

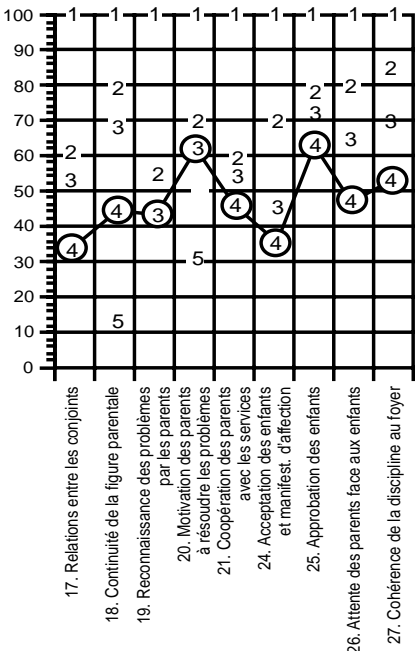

Négligence émotionnelle

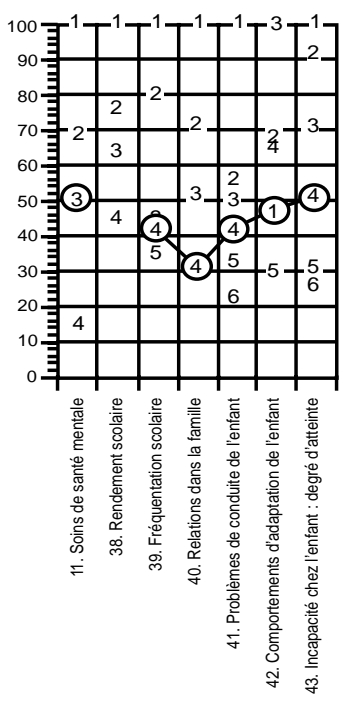

Troubles du comportement 
aux échelles de l'ICBE selon les facteurs et les dimensions théoriques, en utilisant les figures 2 et 3 . Comme pour chacune des 43 échelles de l'ICBE, les scores moyens obtenus aux facteurs et aux dimensions théoriques permettent de cerner des problèmes présents dans la situation de l'enfant et de sa famille, de même que les points forts.

\section{Détermination des alinéas et des seuils d'intervention}

Pour évaluer la situation de l'enfant et de sa famille dans le cadre de la Loi sur la protection de la jeunesse, certaines questions se présentent d'entrée de jeu. Elles touchent principalement la matérialité, la vulnérabilité de l'enfant, les capacités des parents et du milieu en réponse aux besoins de l'enfant. Ces questions visent d'une part à vérifier s'il y a matière à protection dans la situation signalée et, d'autre part, à relever le ou les alinéas de la Loi sur la protection de la jeunesse qui pourraient être invoqués pour définir la compromission.

Pour répondre à ces questions, une analyse est proposée en utilisant les données du tableau 1. À titre d'exemple, au moment de l'évaluation d'un enfant, des indices laissent penser qu'il peut s'agir d'abus physiques. La consultation du tableau 1 permet de connaître les échelles qui donnent de I'information pertinente pour l'alinéa concerné, soit $38 \mathrm{~g}$ ). Si pour ces échelles, l'enfant et sa famille obtiennent des scores faibles, c'est-à-dire des scores pondérés de gravité se situant sur ou sous le seuil d'intervention, I'hypothèse d'abus physique se voit vérifiée. Se retrouvent au tableau 1, en rapport avec l'alinéa $38 \mathrm{~g}$ ), les échelles 29. Discipline physique abusive, 30. Privation physique délibérée (du boire et du manger), 31. Restrictions physiques excessives. Les renseignements recueillis relativement à ces différentes échelles rassemblent rapidement les informations touchant cet aspect de la protection. D'autres échelles peuvent aussi présenter une certaine pertinence et il est toujours possible d'élargir l'analyse pour y inclure les éléments par facteurs ou par dimensions théoriques. N'oublions pas toutefois qu'en regroupant les échelles par facteurs et dimensions théoriques, certaines échelles qu'il est important de considérer dans l'évaluation du bien-être de l'enfant sont exclues $^{5}$.

$\mathrm{Si}$, en d'autres cas, il n'existe pas d'hypothèse précise cernant les éléments présents dans la situation de l'enfant et de la famille, il est possible de déterminer, à partir de la grille des scores pondérés de gravité (figure 1), les échelles qui obtiennent des scores sur et sous le seuil d'intervention et de vérifier par la suite, au tableau 1 , à quel(s) alinéa(s) peuvent s'associer ces échelles.

5. Les échelles $6,13,22,23,28,35$ et 36 ne sont pas représentées dans les facteurs et les dimensions théoriques. 


\section{CONCLUSION}

Les difficultés liées à l'exercice des responsabilités parentales pouvant affecter le bien-être de l'enfant s'avèrent l'un des problèmes les plus importants auxquels notre société doit faire face. Plusieurs rapports gouvernementaux du Québec en font mention, tels que Le rapport de la Commission Rochon, Un Québec fou de ses enfants, La protection sur mesure, un projet collectif, La protection de la jeunesse, plus qu'une loi, La politique de santé et de bienêtre.

L'évaluation de ces problèmes touchant le bien-être de l'enfant en contexte familial est très complexe. D'ailleurs, les instruments pour supporter ces évaluations ont une portée plutôt restreinte et ne sont pas adaptés en contexte québécois. Pourtant, le bien-être de l'enfant constitue une priorité de notre société qui recherche de plus en plus les moyens pour prévenir et corriger les situations qui pourraient lui porter atteinte.

L'élasticité de la définition de bien-être de l'enfant en relation avec l'exercice des responsabilités parentales et la limite des connaissances actuelles de ces problèmes laissent place à une variation dans les jugements que portent les intervenants sur la gravité de ces problèmes et de ces conséquences. Rendre plus objectives les évaluations faites dans ce domaine est un défi à relever.

L'inventaire concernant le bien-être de l'enfant en relation avec l'exercice des responsabilités parentales est un instrument qui répond, par ses qualités métrologiques, à des besoins d'objectivité et d'uniformité chez les intervenants. Il assure une exploration systématique des facettes du bien-être de I'enfant et de l'actualisation des responsabilités parentales. Il est un soutien à l'observation, à l'évaluation, à l'analyse, au diagnostic et à l'intervention. Il ne remplace pas le jugement du professionnel, mais il n'en demeure pas moins un outil pertinent dans tout le processus de l'intervention psychosociale à différentes étapes. II doit être utilisé de façon rigoureuse et judicieuse pour lui assurer et lui conserver toute sa crédibilité. 


\section{Références bibliographiques}

ABIDIN, R.R. et W.T. BURKE (1982). Parenting Stress Index, Charlottesville: Institute of Clinical Psychology, University of Virginia.

ACHENBACK, T.M. (1981). Child Behavior Checklist. Burlington: Family and Community Psychiatry, University of Vermont.

Alderton, R.R. et B.A. HODINOT (1968). Children's Pathology Index. Downsview (Ontario): Sandringham Professional Building.

AlPERN, G.D., T.J. BOLl et M.S. SHEARER (1980). Developmental Profile II. Colorado: Psychological Development Publications.

BECK, D.F. et M.S. JONES, (1980). Family Service Association Follow-Up Questionnaire. Milwaukee: Family Service of America.

BOUCHARD, C. (1991). Un Québec fou de ses enfants, rapport du groupe de travail pour les jeunes. Gouvernement du Québec.

CALDWELL, B.M. et R.H. BRADLEY (1978). Home observation for measurement of the environment (HOME) inventory. Little Rock: Center for child development and education, University of Arkansas at Little Rock.

COMmISSION ROCHON (1988). Rapport de la Commission d'enquête sur les services de santé et les services sociaux. Québec, Gouvernement du Québec.

EdWARDS, A.L. (1982). Techniques of Attitude Scale Construction. Second Edition, New York: Irvington.

EISEN, M., C.A. DONALD, J.E. WARE et R.H. BroOK (1980). Health Insurance Study (HIS) - Child Health Status Measures. Santa Monica (California): Rund Corporation.

EPSTEIN, A.S. (1980). Knowledge Scale. Ypsilanti (Michigan): High/Scope Educational Research Foundation.

GILBERT, N. et J.G. SAVARD (1978). Statistiques. Montréal : Éditions Études vivantes.

JASMIN, H. (1992). Plus qu'une loi. Rapport du groupe de travail sur l'évaluation de la Loi sur la protection de la jeunesse. Gouvernement du Québec.

Koss, M., H. Hatry, A. Millar et T. VAN Houten (1979). Client Outcome Questionnaire. Washington, DC: The Urban Institute.

MAGURA, S. et E.S. MOSES (1986). Outcome Measures for Child Welfare Services. Washington, DC: Child Welfare League of America.

MINISTÈRE DE LA SANTÉ ET DES SERVICES SOCIAUX (1991). La protection sur mesure, un projet collectif. Rapport du groupe de travail sur I'application des mesures de protection de la jeunesse. Gouvernement du Québec.

MINISTÈRE DE LA SANTÉ ET DES SERVICES SOCIAUX (1990). Manuel de référence sur la Loi sur la protection de la jeunesse. Gouvernement du Québec.

Ministère de LA SANTÉ ET DES SERVICES SOCIAUX (1989). Pour améliorer la santé et le bien-être au Québec. Orientations, Gouvernement du Québec.

POlANSKY, N.A., M.A. Chalmers, E. BUTtenWIESER et D. WILliams (1981). Childhood Level of Living Scale (urban version). Athens : School of Social Work, University of Georgia.

QuAY, H.C. et D.R. Peterson (1979). Behavior Problem Checklist. New Brunswick, NJ: School of Professional Psychology, Rutgers University.

TRUDEL, R. et R. ANTONIUS (1991). Méthodes quantitatives appliquées aux sciences humaines. Montréal: Centre éducatif et culturel. 
VÉZINA, A. et R. BRADET (1990). Validation québécoise de l'inventaire concernant le bien-être de l'enfant en relation avec l'exercice des responsabilités parentales. Rapport de recherche. Centre de recherche sur les services communautaires, Université Laval.

VÉZINA, A. et D. PElletier (1992). Un support au diagnostic et à l'intervention. Rapport de recherche. Centre de recherche sur les services communautaires. Université Laval. 
ANNEXE

\section{ÉCHELLE 2 DE L'ICBE: NUTRITION, RÉGIME ALIMENTAIRE}

Cette échelle porte sur la quantité et la qualité des aliments disponibles et fournis à l'enfant ainsi que sur les conséquences d'une alimentation inadéquate. Deux aspects sont pris en considération pour juger de la qualité des aliments: 1. I'équilibre alimentaire (considéré moins sérieusement) et 2. la salubrité (considérée plus sérieusement). La quantité des aliments fournis est mesurée en fonction des aliments préparés et disponibles pour les enfants.

Les aspects mesurés dans cette échelle sont indépendants du caractère délibéré de la participation des parents dans la situation inadéquate. II s'agit strictement de mesurer si les besoins nutritionnels de l'enfant sont satisfaits. Cependant, les causes du problème peuvent être rapportées dans d'autres échelles (p. ex. : la privation délibérée de nourriture à l'échelle 30, une mauvaise gestion financière à l'échelle 15).

\section{Adéquat}

- Les enfants reçoivent des repas réguliers et suffisants, ce qui satisfait aux conditions de base d'un régime alimentaire équilibré.

\section{Acceptable}

- Les repas préparés sont souvent déséquilibrés sur le plan nutritionnel et à l'occasion sont sautés.

- Les enfants préparent eux-mêmes leur nourriture ou doivent compléter leur régime alimentaire à l'extérieur du foyer.

\section{Légèrement inadéquat}

- Les repas sont irréguliers et souvent ne sont pas préparés, mais, habituellement, au moins un repas par jour est fourni à l'enfant.

- Souvent, les enfants se servent eux-mêmes à manger; parfois, ils ne trouvent que des aliments en quantité insuffisante dont la valeur nutritive est inadéquate.

\section{Modérément inadéquat}

- Il y a des périodes de plusieurs jours pendant lesquelles les repas n'ont pas été fournis.

- Il n'y a pratiquement aucune nourriture à la maison et/ou les enfants sont incapables de se nourrir eux-mêmes.

- À l'occasion, les enfants mangent de la nourriture avariée ou des choses non comestibles.

- Les enfants peuvent être affamés, mais ne souffrent d'aucune maladie réelle.

\section{Sérieusement inadéquat}

- Les enfants présentent des symptômes cliniques de malnutrition, de déshydratation ou d'empoisonnement alimentaire.

- L'attention médicale ou la réhabilitation sur le plan de la diète est requise.

- L'hospitalisation n'est pas requise pour des raisons médicales, même si l'enfant peut être temporairement hospitalisé par mesure de protection.

\section{Sévèrement inadéquat}

- La malnutrition, la déshydratation (sévères pertes de poids, anémie, etc.) ou encore l'empoisonnement (fièvre,vomissements, etc.) sont si graves que les enfants doivent être hospitalisés.

\section{$X$ Information insuffisante}

Z Ne s'applique pas 